\title{
Enhanced X-ray variability from V1647 Ori, the young star in outburst illuminating McNeil's Nebula
}

\author{
N. Grosso ${ }^{1}$, J. H. Kastner ${ }^{2}$, H. Ozawa ${ }^{1}$, M. Richmond ${ }^{2}$, T. Simon ${ }^{3}$, \\ D. A. Weintraub ${ }^{4}$, K. Hamaguchi ${ }^{5,6}$, and A. Frank ${ }^{7}$ \\ ${ }^{1}$ Laboratoire d'Astrophysique de Grenoble, Université Joseph-Fourier, Grenoble, 38041, France \\ e-mail: Nicolas.Grosso@obs.ujf-grenoble.fr \\ 2 Rochester Institute of Technology, Rochester, New York 14623-5604, USA \\ 3 Institute for Astronomy, Honolulu, Hawaii 96822, USA \\ 4 Vanderbilt University, Nashville, Tennessee 37235, USA \\ 5 NASA/Goddard Space Flight Center, Greenbelt, Maryland 20771, USA \\ ${ }^{6}$ National Research Council, 500 Fifth Street, NW, Washington, DC 20001, USA \\ 7 University of Rochester, Rochester, New York 14627-0171, USA
}

Received 14 October 2004 / Accepted 11 March 2005

\begin{abstract}
We report a 38 ks X-ray observation of McNeil's Nebula obtained with XMM-Newton on 2004 April 4. V1647 Ori, the young star in outburst illuminating McNeil's Nebula, is detected with XMM-Newton and appears variable in X-rays. We investigate the hardness ratio variability and time variations of the event energy distribution with quantile analysis, and show that the large increase of the count rate from V1647 Ori observed during the second half of the observation is not associated with any large plasma temperature variations as for typical X-ray flares from young low-mass stars. X-ray spectral fitting shows that the bulk $(\sim 75 \%)$ of the intrinsic X-ray emission in the $0.5-8 \mathrm{keV}$ energy band comes from a soft plasma component, with $k T_{\text {soft }}=0.9 \mathrm{keV}(0.7-1.1 \mathrm{keV}$, at the $90 \%$ confidence limit), reminiscent of the X-ray spectrum of the classical T Tauri star TW Hya, for which X-ray emission is believed to be generated by an accretion shock onto the photosphere of a low-mass star. The hard plasma component, with $k T_{\text {hard }}=4.2 \mathrm{keV}(3.0-6.5 \mathrm{keV})$, contributes $\sim 25 \%$ of the total X-ray emission, and can be understood only in the framework of plasma heating sustained by magnetic reconnection events. We find a hydrogen column density of $N_{\mathrm{H}}=4.1 \times 10^{22} \mathrm{~cm}^{-2}\left(3.5-4.7 \times 10^{22} \mathrm{~cm}^{-2}\right)$, which points out a significant excess of hydrogen column density compared to the value derived from optical/IR observations, consistent with the picture of the rise of a wind/jet unveiled from ground optical spectroscopy. The X-ray flux observed with XMM-Newton ranges from roughly the flux observed by Chandra on 2004 March 22 (i.e. 10 times greater than the pre-outburst X-ray flux) to a value two times greater than that caught by Chandra on 2004 March 7 (i.e. 200 times greater than the pre-outburst X-ray flux). The X-ray variability of V1647 Ori in outburst is clearly enhanced. We have investigated the possibility that V1647 Ori displays a periodic variation in X-ray brightness as suggested by the combined Chandra+XMM-Newton data set. Assuming that the X-ray flux density is periodic, the folding of the two Chandra observed X-ray flux densities with the XMM-Newton ones leads to three periodic X-ray light curve solutions. Our best period candidate is 0.72 day, which corresponds to the time scale of the Keplerian rotation at a distance of 1 and 1.4 stellar radius for a one solar mass star aged of 0.5 and $1 \mathrm{Myrs}$, respectively. We propose that the emission measure, i.e. the observed X-ray flux, is modulated by the Keplerian rotation of the inner part of the V1647 Ori accretion disk.
\end{abstract}

Key words. stars: individual: V1647 Ori - stars: pre-main sequence - X-rays: stars

\section{Introduction}

At the end of January 2004, a new bright fan-shaped nebula was discovered serendipitously (McNeil 2004) between M 78/NGC 2068 and the region of HH 24-26. These two well known star-forming regions of the L1630 dark cloud are located in the northern part of the Orion B giant molecular cloud, at a distance of $\sim 400 \mathrm{pc}$ (Anthony-Twarog 1982). At the apex of McNeil's Nebula lies the young stellar object (YSO) IRAS 05436-0007 = 2MASSJ 05461313-0006048
(Clark 1991), associated with a faint $I$-band source (Eislöffel $\&$ Mundt 1997) detected in the (sub)millimeter (1.3 mm source LMZ12 of Lis et al. 1999; and $850 \mu \mathrm{m}$ source OriBsmm 55 of Mitchell et al. 2001). This YSO is now displaying a dramatic optical/IR outburst, which is the origin of the rise of this reflection nebula. Briceño et al. (2004) have constrained the start of this outburst to the beginning of November 2003, and have obtained a light curve in the $I$-band of V1647 Ori (designation of the variable star illuminating McNeil's Nebula; Samus 2004) showing a $\sim 5$ mag brightening in about 4 months. They show 
that the timescale for the nebula to develop is consistent with the light-travel time, indicating that we are observing light from the central source scattered by material in the cometary nebula.

The nature of the V1647 Ori outburst and its connection with other pre-main sequence (PMS) eruptive objects, namely EXors (stellar prototype: EX Lupi; McLaughlin 1946; Herbig et al. 2001), and FUors (stellar prototype: FU Ori; Herbig 1966; Herbig 1977), is still debated at the moment. Reipurth \& Aspin (2004) and McGehee et al. (2004), have noted a resemblance to EXors; whereas Briceño et al. (2004), Ábrahám et al. (2004), Walter et al. (2004), and Kun et al. (2004) have proposed a FUor event. Vacca et al. (2004) have even reported that the near-IR spectrum of V1647 Ori does not appear similar to any known FUor or EXor object. Aspin \& Reipurth (2004) reported an $R$-band observation of V1647 Ori with the Gemini telescope on Mauna Kea. These latest data, obtained on 2004 August 29 (shortly after the object emerged from conjunction with the Sun), indicate that V1647 Ori continues to be in a state of elevated optical/IR emission, and that McNeil's nebula remains bright, and therefore that the duration of the outburst so far exceeds about nine months. Indeed, continued optical/near-IR monitoring are necessary to determine the duration of the outburst, because outbursts last only several months in EXors compared to several decades in FUors. Both types of outburst are thought to be driven by a sudden increase of accretion through a circumstellar disk (e.g., Hartmann \& Kenyon 1996, and references therein), but the distinction between FUors and EXors is still entirely empirical. The interpretation that the outburst event has its origin in accretion processes is supported by high-resolution IR spectra of V1647 Ori showing CO emission lines, likely originating from $\sim 2500 \mathrm{~K}$ gas in an inner accretion disk region where substantial clearing of dust has occurred (Rettig et al. 2005).

The pre-outburst spectral energy distribution (SED) of V1647 Ori from IR to millimeter shows a flat-spectrum source (Ábrahám et al. 2004). This kind of flat SED is usually interpreted in terms of a circumstellar envelope (Kenyon \& Hartmann 1991). Andrews et al. (2004) propose that V1647 Ori is a transition object between a protostar with circumstellar disk plus remnant circumstellar envelope (Class I protostar), and a classical T Tauri star with circumstellar disk (Class II sources). Spitzer observations of V1647 Ori in early March 2004 (Muzerolle et al. 2005) show a factor of 15-20 increase in brightness across the spectrum from the optical to $70 \mu \mathrm{m}$, leading to a bolometric luminosity of $44 L_{\odot}$, i.e. $\sim 15$ times higher than the pre-outburst level.

X-ray imaging spectroscopy observations with Chandra have revealed a factor $\sim 50$ increase in the X-ray count rate from V1647 Ori during its outburst compared to the pre-outburst state (Kastner et al. 2004). The coincidence of a surge in X-ray brightness with the optical/IR outburst demonstrates that strongly enhanced high energy emission from V1647 Ori occurs as a consequence of high accretion rates. The burst of $\mathrm{X}$-rays was most probably generated via star-disk magnetic reconnection events that occurred in conjunction with such mass infall. This process may also launch new collimated outflows, or jets (Goodson et al. 1997). Indeed, before its recent eruption, V1647 Ori had been identified as the exciting source of a chain of extended emission nebulosity that appears to terminate at $\mathrm{HH} 23$, a shock-excited Herbig-Haro object, located $\sim 3^{\prime}$ North from V1647 Ori (Eislöffel \& Mundt 1997; Lis et al. 1999; Reipurth \& Aspin 2004). The presence of these structures suggests that the present optical/IR/X-ray outburst of V1647 Ori may be merely the latest of a series of such events. Another outburst may have occurred about $37 \mathrm{yr}$ before the present event, based on a photograph obtained in Oct. 1966 which shows a similar cometary nebula (Mallas \& Kreimer 1978). No clear evidence for the presence of a molecular outflow has been found in the submillimeter CO spectral line maps (Lis et al. 1999; Andrews et al. 2004). However the $\mathrm{H} \alpha$ line, which has been detected in strong emission, displays a pronounced $\mathrm{P}$ Cygni profile, with an absorption trough reaching velocities up to $600 \mathrm{~km} \mathrm{~s}^{-1}$ (Reipurth \& Aspin 2004), implying significant mass loss in a powerful wind.

We report here an X-ray observation of V1647 Ori in outburst obtained with XMM-Newton, showing enhanced X-ray variability from V1647 Ori. We present in Sect. 2 the $X M M-N e w t o n$ observation and data reduction, we study the variability in Sect. 3, and the X-ray spectrum in Sect. 4. We compare the X-ray flux observed with Chandra and $X M M-N e w t o n$ in Sect. 5, and we discuss in Sect. 6 the origin of the X-ray flux variations observed from V1647 Ori.

\section{XMM-Newton observations and data reduction}

We observed V1647 Ori with XMM-Newton (Jansen et al. 2001) on 2004 April 4. The pointing nominal J2000 coordinates were $\alpha_{\mathrm{J} 2000}=05^{\mathrm{h}} 46^{\mathrm{m}} 13.1, \delta_{\mathrm{J} 2000}=-00^{\circ} 06^{\prime} 04^{\prime \prime} .6$. We used the full frame science mode of the EPIC cameras with medium optical blocking filter. The total exposure times were $\sim 37.0 \mathrm{ks}$ and $\sim 38.7 \mathrm{ks}$ for the EPIC/PN (Strüder et al. 2001) and the two EPIC/MOS spectro-imagers (Turner et al. 2001), respectively. We also acquired optical and UV data with the Optical Monitor (OM) with the following filter sequence: $V, U, B$, and $U V W 1$; however our OM data are severely contaminated by stray light (Mason et al. 2001) from the extended reflection nebula $\mathrm{M} 78$, which is located $\sim 10^{\prime}-\sim 15^{\prime}$ North-East of V1647 Ori, preventing us from using these data at the position of V1647 Ori. We will focus here only on the X-ray detection of V1647 Ori.

The data were reduced using the XMM-Newton Science Analysing System (SAS, version 6.0.0) ${ }^{1}$. The event lists were produced using tasks epchain and emchain. The observation is mainly affected by a background proton flare having a duration of one hour, centered at $1 \mathrm{~h}$ UT on 2004 April 4, and peaking on $\mathrm{PN}^{2}$ to 50-150 count ks ${ }^{-1} \operatorname{arcmin}^{-2}$. Removal of time intervals with flaring background above 10 and 5 count ks arcmin $^{-2}$ for PN and MOS, respectively, leads to effective exposure times of $\sim 33 \mathrm{ks}$ and $\sim 38 \mathrm{ks}$, respectively.

\footnotetext{
${ }^{1}$ For a description of the standard procedures see, e.g., the $X M M$-Newton ABC Guide, Snowden et al. (2004), available at http://heasarc.gsfc.nasa.gov/docs/xmm/abc/

2 Background value obtained from the detector background light curve provided for PN by epchain.
} 
X-ray images were then produced by selecting good events within the fields-of-view of the detectors ${ }^{3}$.

Vignetting corrections were made from exposure maps computed on the corresponding energy bands. Figure 1 shows the tricolour X-ray image of the region surrounding McNeil's Nebula (see for comparison Fig. 2 of Kastner et al. 2004). The X-ray source XMM J054613.1-000604 found by the pipeline is located at $\alpha_{\mathrm{J} 2000}=05^{\mathrm{h}} 46^{\mathrm{m}} 13.2, \delta_{\mathrm{J} 2000}=-00^{\circ} 06^{\prime} 04^{\prime \prime} .1$ with a $1 \sigma$ error box of $0.2^{\prime \prime}$, and displays a count rate of $0.036 \pm 0.001$ count s$^{-1}$ in PN and $0.012 \pm 0.001$ count $^{-1}$ in each MOS detector within the energy band $0.2-12 \mathrm{keV}$. This $\mathrm{X}$-ray source is located at $0.9^{\prime \prime}$ from the 2 MASS position of V1647 Ori. Computing the root-mean-square dispersion of distances between the positions of X-ray sources found by the pipeline and their 2MASS counterpart positions, we found the same offset value, therefore we will consider this XMM-Newton source to be the same X-ray counterpart of V1647 Ori already detected by Chandra (Kastner et al. 2004). The other X-ray sources visible in Fig. 1 are (from left to right): XMM J054619.4-000520, XMM J054618.9-000539, and XMMJ054611.6-000627, which are the X-ray counterparts to SSV $64=\mathrm{LkH} \alpha 301$, GSC2 S020001258, and 2MASS J05461162-0006279, respectively (see Kastner et al. 2004; and Simon et al. 2004).

We selected in each camera X-ray events of the V1647 Ori X-ray source for variability and spectral analysis. For PN, we selected only single and double pixel events (i.e. PATTERN in the 0 to 4 range) with FLAG value equal to zero. Source plus background events were extracted within a circular region using the SAS task eregionanalyse. The extraction position and radius $\left(r=23^{\prime \prime}\right)$ were optimized by this task to maximize the signal-to-noise ratio, taking for the estimation of the background an annular region $\left(r=23^{\prime \prime}-44^{\prime \prime}\right)$ centered on this source and located on the same CCD, where areas illuminated by other X-ray sources were excluded. Background events of this annular region were also extracted.

\section{Variability and quantile analysis}

We limited our variability analysis to the PN observing time interval, where all three EPIC cameras were working together, which allowed us to build directly an EPIC (PN+MOS1+MOS2) light curve. Using the SAS task evselect, we first built two light curves with $1 \mathrm{~s}$ time bins from the source+background and the background event lists (where we kept flaring background time intervals). These light curves begin exactly at the start of the first Good Time Interval (GTI) of the PN aimpoint CCD \#4 (where the source is detected). We rebinned the extracted light curve to a longer time interval with the ftools task lcurve to increase the signal. Then, we subtracted from the source+background light curve the background light curve scaled to the same source extraction area, using the ftools task 1 cmath. We also corrected this source light curve for the observing time lost, for example,

\footnotetext{
${ }^{3}$ We selected single, double, triple, and quadruple pixel events (PATTERN in the 0 to 12 range) for both PN and MOS, plus the FLAG list \#XMMEA_EM for MOS.
}

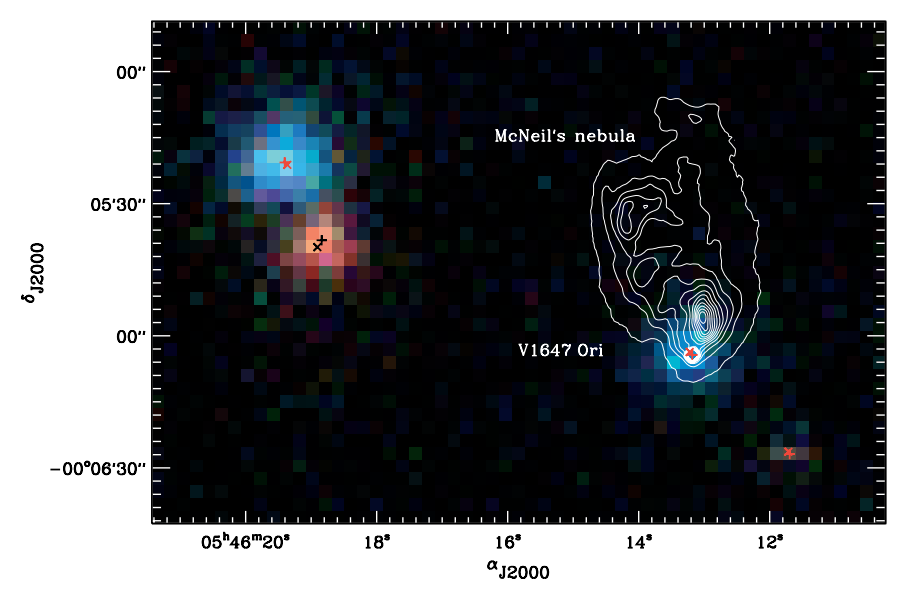

Fig. 1. XMM-Newton/EPIC image of the region surrounding McNeil's Nebula. The three images obtained with PN, MOS1, and MOS2 EPIC cameras on 2004 April 4 were added after correction from vignetting. The size of the image pixel is $3^{\prime \prime}$. This image has red, green, and blue colour coding for X-ray photons in the $0.5-1.5,1.5-2.4$, and $2.4-8.0 \mathrm{keV}$ energy bands, respectively (see algorithm of Lupton et al. 2004). For comparison purposes we overlay a $R$-band contour map of the nebula obtained with VLT/FORS2 on 2004 February 18. $\mathrm{X}$-ray sources and 2MASS sources are marked with " $\times$ " and "+" respectively. A bright embedded X-ray source is spatially coincident with V1647 Ori at the apex of McNeil's nebula.

due to the triggering of counting mode during high flaring background periods, when the count rate exceeded the detector telemetry limit. From the GTI extension, we computed with an IDL routine for each time bin the ratio between the time bin length and the observing time lost in this time bin, and we multiplied count rate and error by this linear correction factor. Finally, light curves of the three detectors were summed to produce the EPIC light curve.

The upper panel of Fig. 2 shows the EPIC X-ray light curve of V1647 Ori in the energy band from 0.5 to $8.0 \mathrm{keV}$ binned to $1000 \mathrm{~s}$. The source is variable, with a low level in count rate during the first half of the observation, varying between $\sim 0.025$ and $\sim 0.005$ count s$^{-1}$ (note that the decrease of count rate around $1 \mathrm{~h}$ UT cannot be a consequence of the high flaring background because we have corrected for the losses of observing time due to triggering of counting mode). This low level was then followed by a much higher level of count rate during the second half of the observation. This latter phase consisted of a slow $(\sim 4 \mathrm{~h})$ rise with an abrupt jump to a peak at $\sim 0.13$ count $^{-1}$, and then a faster $(\sim 1 \mathrm{~h})$ decay from the peak of $\sim 0.13$ count $^{-1}$ back down to the general level of enhanced emission seen from $5 \mathrm{~h}$ onwards. A similar time behaviour is visible both in the soft energy band $(S=0.5-2.8 \mathrm{keV})$ and in the hard energy band $(H=2.8-8 \mathrm{keV})$. To quantify possible softening or hardening of the source events with time, we use the standard hardness ratio method by computing $H R=(H-S) /(H+S)$ (where the hardness ratio errors are computed using Gaussian error propagation). The hardness ratios for the first and the second halves of the observation are $H R=-0.29 \pm 0.10$ and $0.12 \pm 0.03$, respectively (see the lower panel of Fig. 2). 


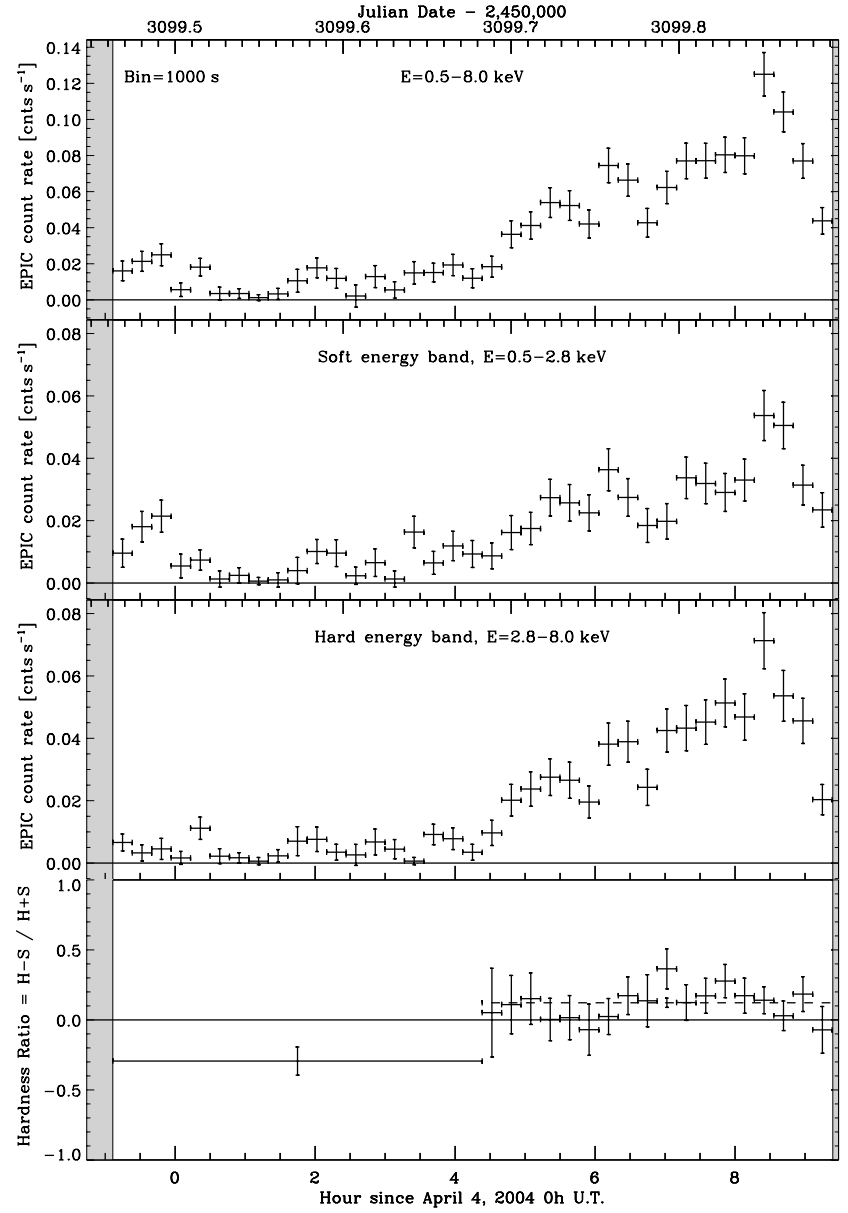

Fig. 2. XMM-Newton/EPIC background subtracted X-ray light curves of V1647 Ori. The upper panel shows the EPIC (PN+MOS1+MOS2) $\mathrm{X}$-ray light curve of V1647 Ori with one sigma error bars in the energy band from 0.5 to $8.0 \mathrm{keV}$. The grey stripes indicate the small observational time intervals before the start and the end of the PN exposure where only the two MOS detectors were observing. The middle panels show the soft $(S=0.5-2.8 \mathrm{keV})$ and hard $(H=2.8-8 \mathrm{keV})$ band $\mathrm{X}$-ray light curves. The lower panel shows the variation of the corresponding hardness ratio. The dashed line indicates the hardness ratio for the second half of the observation.

A least-square fit assuming constant hardness ratio leads to $\chi^{2}=15.4$ (with one degree of freedom), with a probability ${ }^{4}$ of $Q \sim 10^{-4}$. This fit therefore excludes, at the $99.99 \%$ confidence level, the possibility that the hardness ratio remained constant during the XMM-Newton observation. We conclude that the X-ray emission from V1647 Ori hardened during the second half of the observation.

However during the second half of the observation, there is no correlation between the observed variations of the full energy band light curve and the hardness ratio. The hardness ratio values computed within time bins of $1000 \mathrm{~s}$ (see the lower panel of Fig. 2) are consistent with a constant level (with a probability of $89 \%$ ). In particular, the rise and the decay of the light

\footnotetext{
${ }^{4}$ Here, $Q$ is the probability that the best-fit model matches the data, given the value of $\chi^{2}$. Values of $Q<0.05$ indicate that the best-fit model is a poor fit, while $Q \sim 1$ indicates the fit is overconstrained. Optimal values of $Q$ are $\sim 0.5$.
}

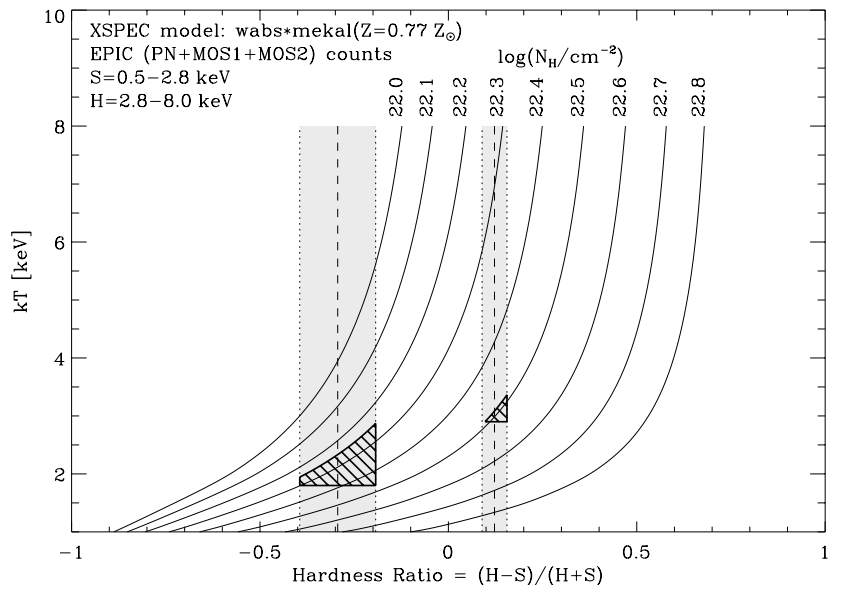

Fig. 3. Comparison between the observed hardness ratios and absorbed one temperature plasma models. The left and right hand vertical grey stripes indicate the hardness ratio values of the first and the second halves of the observation, respectively, measured with EPIC (see lower panel of Fig. 2). The curves give plasma temperature versus hardness ratio for a fixed value of the hydrogen column density. The dashed areas show the constraints on the model parameters when combining the results from the quantile analysis of PN data (see lower panel of Fig. 4) with hardness ratios measured with EPIC.

curve do not correspond to any hardening and softening of the spectrum, whereas such a correspondence is usually observed in typical X-ray flares from young low-mass stars that are associated with the heating and the cooling phases of the emitting plasma (e.g., Tsuboi et al. 1998; Imanishi et al. 2003); in such cases, the absorbing column density is observed to remain constant.

Figure 3 shows a comparison of the hardness ratio values measured with EPIC for the first and the second halves of the observation with absorbed one temperature plasma models computed with XSPEC (version 11.3.0) ${ }^{5}$. We cannot disentangle possible changes in absorbing column density from changes in plasma temperature with only one hardness ratio. The usual improvement would be to introduce three consecutive energy bands, which would allow the computation of two hardness ratios: the first built on soft and medium energy bands, sensitive to the absorbing column density; the second built on medium and hard energy bands, sensitive to the plasma temperature. However the total number of counts is too limited to permit us to obtain two hardness ratios with small enough error bars to allow a temporal study. To improve our analysis, we investigate changes in the energy of the source X-ray photons collected by PN from quantile analysis, as proposed by Hong et al. (2004).

To avoid signal-to-noise variations, we define variablelength time bins in which the total number of net counts collected by PN is constant, and equal to 200 net counts per time bin. This leads to 5 time bins(where bin \#1 corresponds to the first half of the observation) and thereby allows a variability

5 XSPEC wabs $\times$ mekal model, with global metallicity abundance $Z=0.77 Z_{\odot}$ (obtained from spectral fitting; Sect. 4), using the PN, MOS1, and MOS2 redistribution matrix files and ancillary response files appropriate for V1647 Ori (on-axis source). 

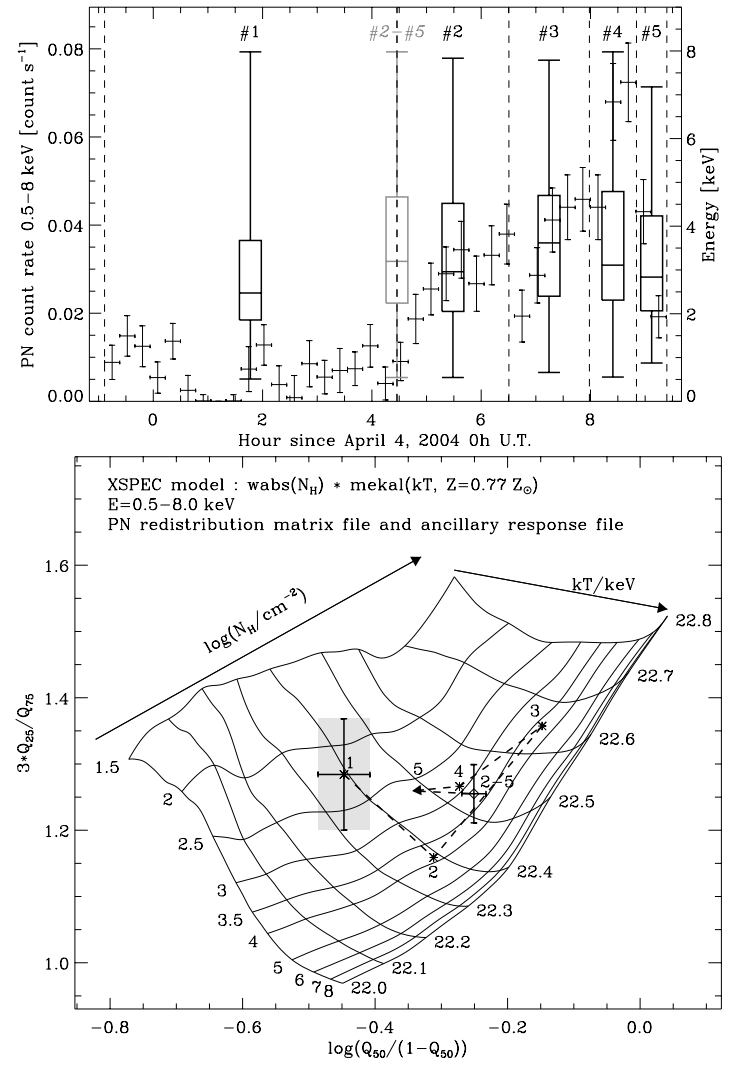

Fig. 4. Quantile analysis of the energy of the X-ray photons of V1647 Ori collected by EPIC/PN. The upper panel shows the EPIC/PN light curve of V1647 Ori in the $0.5-8.0 \mathrm{keV}$ energy band. Vertical dashed lines define variable-length time bins numbered from \#1 to \#5 where PN collected 200 net counts. For each time bin, the box plot indicates the energy distribution of the source (the vertical line and the box indicate the energy range and the quartiles/median, respectively) corrected from background (except minimum and maximum values) using the Hong et al. (2004) method. The grey box plot corresponding to bin \#2-\#5 indicates the energy distribution of the entire second half of the observation. The lower panel shows the X-ray colour-colour diagram based on median and the ratio of two quartiles (Hong et al. 2004). The numbered asterisks mark the X-ray colours corresponding to the time bins defined in the upper panel. The diamond marks the X-ray colours of the second half of the observation. Typical one sigma error bars are shown for comparison.

study. Following Hong et al. (2004), we determine for each time bin median $\left(E_{50 \%}\right)$ and quartile $\left(E_{25 \%}, E_{75 \%}\right)$ energies of the source+background X-ray photons collected by PN, corrected for the background X-ray photons (see upper panel of Fig. 4$)$; and associated quantiles, $Q_{\mathrm{x} \%}=\left(E_{\mathrm{x} \%}-E_{\text {low }}\right) /\left(E_{\mathrm{up}}-\right.$ $\left.E_{\text {low }}\right)$, with $E_{\text {low }}=0.5 \mathrm{keV}$ and $E_{\text {up }}=8 \mathrm{keV}$. Then, we compute the two X-ray colours proposed by Hong et al. (2004): $x \equiv$ $\log \left(Q_{50 \%} /\left(1-Q_{50 \%}\right)\right)$, and $y \equiv 3 \times Q_{25 \%} / Q_{75 \%}$. These definitions of X-ray colours lead to a unique correspondance between the X-ray colour pair $(x, y)$ and plasma parameters $\left(N_{\mathrm{H}}, T\right)$, where $N_{\mathrm{H}}$ is the absorbing hydrogen column density, and $T$ the plasma temperature. The lower panel of Fig. 4 shows the reference mapping of the $x, y$ space-parameter that we obtained by simulating with XSPEC absorbed one-temperature plasma spectra from an on-axis source observed with PN. This grid allows a direct estimate of $\log N_{\mathrm{H}}$ and $k T$ from $x$ and $y$. Based on 10000 trials of synthetic spectra computed from a model with parameters $\log N_{\mathrm{H}}=22.5, k T=3.0 \mathrm{keV}, Z=0.77 Z_{\odot}$ (see Sect. 4 and model \#1 in Table 1), and 200 counts, we found that the errors on $x$ and $y$ colours are distributed normally. In the lower panel of Fig. 4, we plot one sigma error bars on $x$ and $y$ for a single (200 net count) time bin.

For bin \#1, taking into account the one sigma uncertainties on the X-ray colours, we find $\log N_{\mathrm{H}} \sim 22.4(22.25-22.5)$, and $k T \sim 2.2 \mathrm{keV}(1.8-2.8 \mathrm{keV})$. Over the course of the entire observation, the $y$ colour varied by less than the one sigma uncertainty for a single time bin. From bin \#1 to bins \#3 and \#4, there is a variation of the $x$ colour greater than the one sigma error bar. However, due to the large error on the $y$ colour, this variation in $x$ colour implies no significant variation of the hydrogen column density and/or the plasma temperature. During the second half of the observation, we find $\log N_{\mathrm{H}} \sim$ $22.53(22.49-22.57)$ and $k T \sim 3.2 \mathrm{keV}(2.9-3.7 \mathrm{keV})$; because $\sim 80 \%$ of the photons were detected in this latter half of the observation, the uncertainties on the quantile values obtained for the second half of the observation are smaller by a factor $\sim 2$ than the uncertainties in the first half of the observation. Therefore, there appears to be a hint of an increase in plasma temperature during the second half of the observation.

In Fig. 3, we combine these constraints on the hydrogen column density and the plasma temperature of the first and the second halves of the observation with the hardness ratio values obtained previously. We conclude that the hardening of the spectrum can be explained by a rather small increase of the plasma temperature from $\sim 2 \mathrm{keV}$ to $\sim 3 \mathrm{keV}$, combined with a small increase of the absorbing column density from $\log N_{\mathrm{H}} \sim 22.3$ to $\log N_{\mathrm{H}} \sim 22.5$.

It appears overall that the large increase of the count rate is not associated with any large variations of the temperature. This lack of strong correlation between X-ray flux and temperature suggests that we are perhaps also observing variations in the emission measure of the $\mathrm{X}$-ray-emitting plasma. The variability observed with XMM-Newton and that measured previously with Chandra on 2004 March 7 and 22 will be compared further in Sect. 5 to investigate this possibility.

\section{Spectral analysis}

We created source and background spectra with associated redistribution matrix files and ancillary response files using the SAS task especget applied on the same source and background extraction region used for the light curve, and we binned the spectra to 15 counts per spectral bin using the ftools task grppha. Figure 5 shows PN, MOS1, and MOS2 spectra of the whole observation after suppression of high flaring background periods. The spectrum is clearly absorbed, with only a few counts detected by PN below $1 \mathrm{keV}$ in energy, whereas PN detected counts up to $9 \mathrm{keV}$ in energy. A prominent line is visible in PN spectra around $6.7 \mathrm{keV}$, corresponding to the Fe XXV triplet emission line. We performed spectral modeling with XSPEC (version 11.3.0) using a multi-temperature plasma (mekal) model (Kaastra et al. 1996) with intervening absorption (as represented by the wabs model, which uses 
Table 1. Best fit parameters of EPIC spectrum models with their errors at the $90 \%$ confidence levels $\left(\Delta \chi^{2}=2.71\right.$; corresponding to $\sigma=1.64$ for Gaussian statistics). For PN, MOS1, MOS2, the effective exposures after suppression of time intervals with high levels of flaring background are $33.2,37.7$, and $38.1 \mathrm{ks}$, and there are in the source extraction region 1264, 408, 408 counts, respectively ( $20 \%$ of these counts come from the background).

\begin{tabular}{|c|c|c|c|c|c|c|c|c|c|c|c|}
\hline \multirow[b]{2}{*}{$\begin{array}{c}\text { Model } \\
\#\end{array}$} & \multirow[b]{2}{*}{$\begin{array}{c}N_{\mathrm{H}, 22} \\
{\left[\mathrm{~cm}^{-2}\right]}\end{array}$} & \multirow[b]{2}{*}{$\begin{array}{c}Z \\
{\left[Z_{\odot}\right]}\end{array}$} & \multicolumn{2}{|c|}{ Temperature } & \multicolumn{2}{|c|}{ Emission measure $^{a}$} & \multicolumn{2}{|c|}{$6.4 \mathrm{keV} \mathrm{Fe} \mathrm{line}{ }^{b}$} & \multirow[b]{2}{*}{$\chi_{v}^{2}(v)^{c}$} & \multirow[b]{2}{*}{$\begin{array}{c}Q^{d} \\
{[\%]}\end{array}$} & \multirow{2}{*}{ 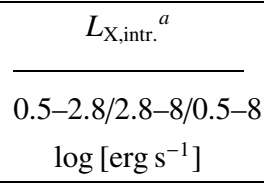 } \\
\hline & & & $\begin{array}{l}T_{\text {soft }} \\
\quad[\mathrm{keV}\end{array}$ & $T_{\text {hard }}$ & $E M_{\text {soft }}^{54}$ & {$\left[\mathrm{~cm}^{-3}\right]$} & $\begin{array}{c}N_{\mathrm{ph},-7} \\
{\left[\mathrm{~cm}^{-2} \mathrm{~s}^{-1}\right]}\end{array}$ & $\begin{array}{r}E W \\
{[\mathrm{eV}]}\end{array}$ & & & \\
\hline 1 & $2.9_{-0.4}^{+0.5}$ & $0.8_{-0.3}^{+0.5}$ & & $3.0_{-0.6}^{+0.9}$ & $\ldots \ldots$ & $0.7 \pm 0.1$ & & $\cdots$ & $1.11(129)$ & 19 & 30.830 .531 .0 \\
\hline 2 & $4.1 \pm 0.6$ & $0.8_{-0.3}^{+0.4}$ & $0.9 \pm 0.2$ & $4.3_{-1.3}^{+2.2}$ & $1.2_{-0.6}^{+1.3}$ & $0.4_{-0.1}^{+0.2}$ & & & $0.97(127)$ & 56 & 31.430 .531 .5 \\
\hline 3 & $4.1 \pm 0.6$ & $0.8_{-0.3}^{+0.4}$ & $0.9 \pm 0.2$ & $4.2_{-1.3}^{+2.4}$ & $1.3_{-0.6}^{+1.4}$ & $0.4_{-0.1}^{+0.2}$ & $4.3_{-4.3}^{+4.5}$ & 109 & $0.96(126)$ & 60 & 31.430 .531 .5 \\
\hline
\end{tabular}

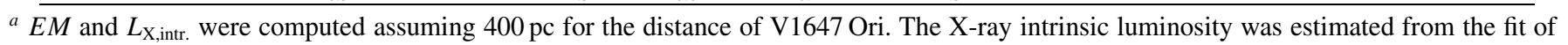
the PN data.

${ }^{b}$ Energy and width fixed to $6.4 \mathrm{keV}$ and $12 \mathrm{eV}$, respectively.

${ }^{c} v$ is the degree of freedom, and $\chi_{v}^{2}$ is the reduced Chi-square (i.e. the Chi-square divided by the degree of freedom).

${ }^{d}$ The $Q$-value is the probability that one would observe the Chi-square value, or a larger value, if the assumed model is true, and the best-fit model parameters are the true parameter values.

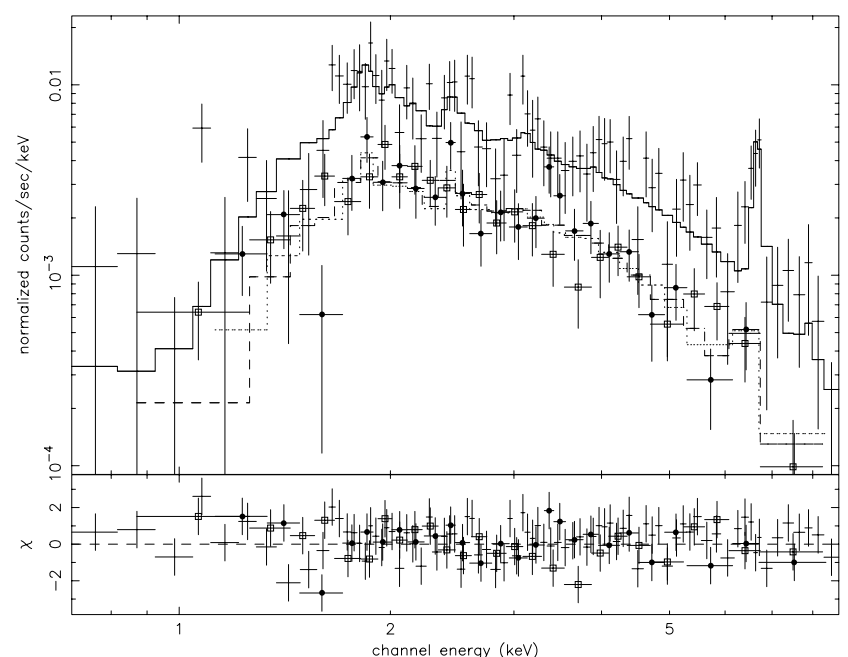

Fig. 5. XMM-Newton X-ray spectra and our best fit models of V1647 Ori. The upper panel shows the data binned to 15 counts in each spectral bin, and our best fit models with an absorbed two-temperature optically thin plasma (see model \#2 in Table 1). Continuous line, dashed line plus squares, and dotted line plus disks, stand for PN, MOS1, and MOS2, respectively. The lower panel shows the residuals of the fit, in units of the uncertainties in the individual data points. The Fe XXV emission line at $6.7 \mathrm{keV}$ is clearly visible.

Morrison \& McCammon 1983, cross-sections). Table 1 gives the best fit parameters that we found for each statistically acceptable model (i.e. each model leading to a fit with $Q$-value greater than 5\%), with the corresponding $90 \%$ confidence level intervals.

The absorbed one-temperature plasma model (wabs $\times$ mekal) leads to $N_{\mathrm{H}}=2.9 \times 10^{22} \mathrm{~cm}^{-2}$ $\left(2.5-3.4 \times 10^{22} \mathrm{~cm}^{-2}\right), k T=3.0 \mathrm{keV}(2.4-3.8 \mathrm{keV})$, with $Z=0.8 Z_{\odot}\left(0.4-1.2 Z_{\odot}\right)$, i.e. column density and temperature values consistent with the ones previously determined from our quantile analysis in Sect. 3. This quantile analysis remains
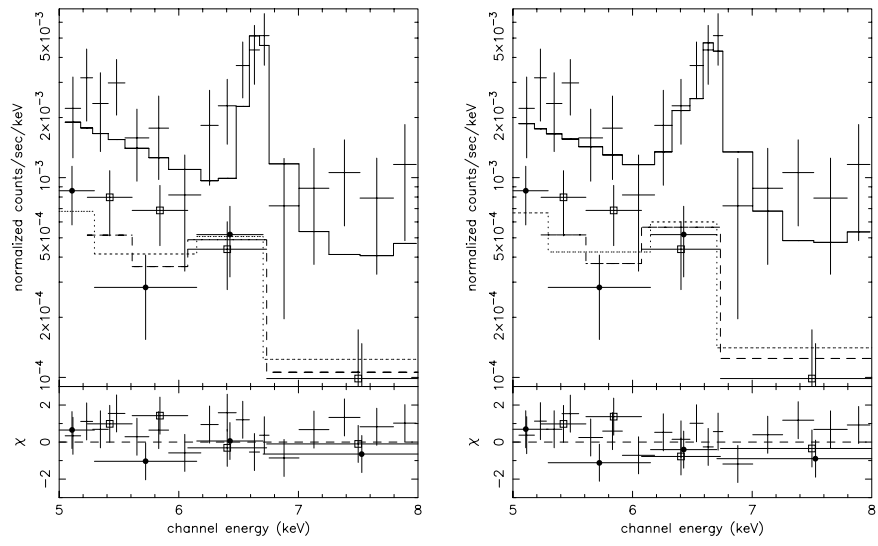

Fig. 6. Fit around the $6.7 \mathrm{keV}$ iron line region. The left hand panel shows an enlargement of the absorbed two-temperature model (see model 2 in Table 1) around the $6.7 \mathrm{keV}$ iron line, using a linear energy scale. The right hand panel shows the same energy area adding a Gaussian line fitting the $6.4 \mathrm{keV}$ neutral iron line (see model 3 in Table 1). Symbols are identical to the ones of Fig. 5.

the only time-dependent spectral analysis possible with this data set due to the small number of total counts.

We obtain a fit with a better probability $(Q=56 \%)$ when we add a second temperature component. Therefore, we consider this model (\#2 in Table 1) as our best fit. The bulk ( 75\%) of the emission measure in the $0.5-8 \mathrm{keV}$ energy band, and therefore most of the intrinsic X-ray emission, arises with a soft plasma component, with $k T_{\text {soft }}=0.9 \mathrm{keV}(0.7-1.1 \mathrm{keV})$; whereas a hard plasma component, with $k T_{\text {hard }}=4.2 \mathrm{keV}$ (3.0-6.5 keV) contributes $\sim 25 \%$ of the total X-ray emission. For this two-temperature model we find a column density a bit larger than in model \#1, with $N_{\mathrm{H}}=4.1 \times 10^{22} \mathrm{~cm}^{-2}$ $\left(3.5-4.7 \times 10^{22} \mathrm{~cm}^{-2}\right)$.

One or two-temperature plasma models with the hydrogen column density fixed to the lowest value found from optical/IR observations of V1647 Ori during outburst (e.g., $A_{\mathrm{V}}=11 \mathrm{mag}$ 
from Vacca et al. 2004, see discussion below) lead to poor fits $(Q<0.05)$ and, hence, are not acceptable.

The $\mathrm{K} \alpha$ fluorescent line from neutral to low-ionized iron at $6.4 \mathrm{keV}$ was detected by Imanishi et al. (2001) with Chandra from a class I protostar in the $\rho$ Ophiuchi dark cloud during a bright flare, and was interpreted as reflection emission from a face-on accretion disk. We estimate the $90 \%$ confidence limit on the strength of a neutral $6.4 \mathrm{keV}$ iron line in the V1647 Ori spectrum by adding a Gaussian line of $12 \mathrm{eV}$ width at $6.4 \mathrm{keV}$. This will be important to constrain future 2D models involving the heating of plasma and its interaction with nearby circumstellar material. We retrieve exactly the best-fit parameters found for model \#2 (see Table 1), and the Gaussian line at $6.4 \mathrm{keV}$ appears to improve the fit in the $6.7 \mathrm{keV}$ iron line region (see Fig. 6). However, we find that the total photon rate in the line is $N_{\mathrm{ph}}=4.3 \times 10^{-7} \mathrm{~cm}^{-2} \mathrm{~s}^{-1}\left(0-8.7 \times 10^{-7} \mathrm{~cm}^{-2} \mathrm{~s}^{-1}\right)$ so, given this range of uncertainty, we cannot exclude the possibility that there is no $6.4 \mathrm{keV}$ iron line in the XMM-Newton spectrum of V1647 Ori.

Both the temperature and the column density found here with the one-temperature plasma model are consistent with results obtained from the same spectral modeling of Chandra data obtained on 2004 March 7. The large contribution from soft plasma emission to the X-ray spectrum of V1647 Ori revealed by XMM-Newton data is reminiscent of that observed from TW Hya (Kastner et al. 2002). For TW Hya, the soft X-ray emission is believed to be generated via mass accretion from its circumstellar disk, which produces shocks onto the stellar photosphere. However the hard plasma emission of V1647 Ori above $1 \mathrm{keV}$ is too elevated to be explained in terms of accretion shocks onto a low-mass star, and can be understood only in the framework of plasma heating sustained by magnetic reconnection events (e.g., Kastner et al. 2004).

This XMM-Newton measurement of the hydrogen column density, consistent with the previous Chandra estimate, is now well enough constrained to reveal a significant excess of hydrogen column density compared to the value derived from optical/IR observations. Combining $N_{\mathrm{H}} / A_{\mathrm{J}}=5.6 \pm 0.4 \times$ $10^{21} \mathrm{~cm}^{-2} \mathrm{mag}^{-1}$ (Vuong et al. 2003), and $A_{\mathrm{J}} / A_{\mathrm{V}}=0.4008-$ $0.1187 \times\left(R_{\mathrm{V}} / 3.1\right)^{-1}$ (Cardelli et al. 1989), leads to $A_{\mathrm{V}}=$ $\left(N_{\mathrm{H}} / 10^{21}\right) /\left[2.24-0.66 \times\left(R_{\mathrm{V}} / 3.1\right)^{-1}\right]$, where the parameter $R_{\mathrm{V}}$ ranges from the average galactic value of 3.1 to $\sim 5.5$ for a dusty environment. Therefore our measurement, $N_{\mathrm{H}}=$ $3.5-4.7 \times 10^{22} \mathrm{~cm}^{-2}$, is equivalent to $A_{\mathrm{V}}=22.3-29.6 \mathrm{mag}$ and $18.8-25.0 \mathrm{mag}$, for $R_{\mathrm{V}}=3.1$ and 5.5 , respectively. The highest value found for the visual extinction of V1647 Ori in its outburst is $A_{\mathrm{V}}=11 \mathrm{mag}$ (Vacca et al. 2004), which is well below our $90 \%$ confidence interval. We stress that there remains a discrepancy between our lower range estimate and this highest visual extinction for any value of the parameter $R_{\mathrm{V}}$. We conclude that there is an excess of hydrogen column density in the local environment of V1647 Ori of at least $1.5-2.6 \times 10^{22} \mathrm{~cm}^{-2}$, assuming the visual extinction of Vacca et al. (2004), and $R_{\mathrm{V}} \sim 5.5$. The gas-to-dust ratio along the line-of-sight towards V1647 Ori is hence unusual, with more gas than dust towards this object. This is consistent with the picture of the rise of a wind/jet from the central embedded source, which would increase the amount of gas close to central object where the $\mathrm{X}$-ray emission is believed to arise.

\section{Comparison of V1647 Ori X-ray fluxes observed with Chandra and XMM-Newton}

We have updated Fig. 1 of Kastner et al. (2004) by adding IR measurements of Walter et al. (2004), which completes the monitoring of V1647 Ori through 2004 April, and XMM-Newton measurements (see the left hand panel of Fig. 7). XMM-Newton observed serendipitously the region of V1647 Ori on 2003 September 3, with 50 ks exposure, by pointing toward the Herbig-Haro emission complex HH24-26 located about 4' south (Ozawa et al., in preparation). V1647 Ori was not detected. We convert this non-detection (computed on a $\sim 25 \mathrm{ks}$ exposure without flaring background) to a flux density upper limit assuming an absorbed $\left(N_{\mathrm{H}}=5.7 \times\right.$ $\left.10^{22} \mathrm{~cm}^{-2}\right)$ one-temperature $(k T=4.8 \mathrm{keV})$ model with $Z=$ $0.3 Z_{\odot}$, i.e. the same model used by Kastner et al. (2004) to derive flux density from the Chandra detection of V1647 Ori on 2002 November 14. The resulting XMM-Newton upper limit is consistent with the low pre-outburst activity level of V1647 Ori observed by Chandra on 2002 November 14 during a $55.9 \mathrm{ks}$ exposure. This demonstrates that the rise of X-ray activity had not yet occured two months before the optical/IR outburst and strengthens the apparent correlation between the sharp increase in X-ray flux from pre-outburst to outburst and the similar brightening seen in the optical/IR, already noticed by Kastner et al. (2004). Using our best-fit spectral model, we convert EPIC count rate in the $0.5-8 \mathrm{keV}$ energy range (see Fig. 2) to observed X-ray flux density (note that the conversion factor is essentially independent of the number of plasma components). As shown in the right hand panel of Fig. 7, the $\mathrm{X}$-ray flux observed with XMM-Newton ranges from roughly the flux observed by Chandra on 2004 March 22 (i.e. 10 times greater than the pre-outburst X-ray flux) to a peak at a value two times greater than the one caught by Chandra on 2004 March 7 ( 200 times greater than the pre-outburst X-ray flux). The $\mathrm{X}$-ray variability of V1647 Ori in outburst is hence also clearly enhanced, as noted by Kastner et al. (2004).

The right hand panel of Fig. 7 reveals that the X-ray flux density of V1647 Ori during the first half of the XMM-Newton observing period on 2004 April 4 is nearly identical to the flux density of the source during the epoch of the Chandra observations on 2004 March 22. Similarly, the enhanced flux density of V1647 Ori during the second half of the XMM-Newton observing period is an excellent match to the X-ray brightness of the source during the epoch of the Chandra observations on 2004 March 7. The combined Chandra+XMM-Newton data set strongly suggests the possibility that the V1647 Ori shows a periodic variation in X-ray brightness. We have investigated this possibility by folding the Chandra flux measurements with those from XMM-Newton.

For this purpose, we define the following function:

$\mathcal{D}(P) \equiv \sqrt{\sum_{i=1}^{2} \frac{\left(F_{C X O, i}-\tilde{F}_{X M M, i, P}\right)^{2}}{\sigma_{X M M, i, P}^{2}}}$ 

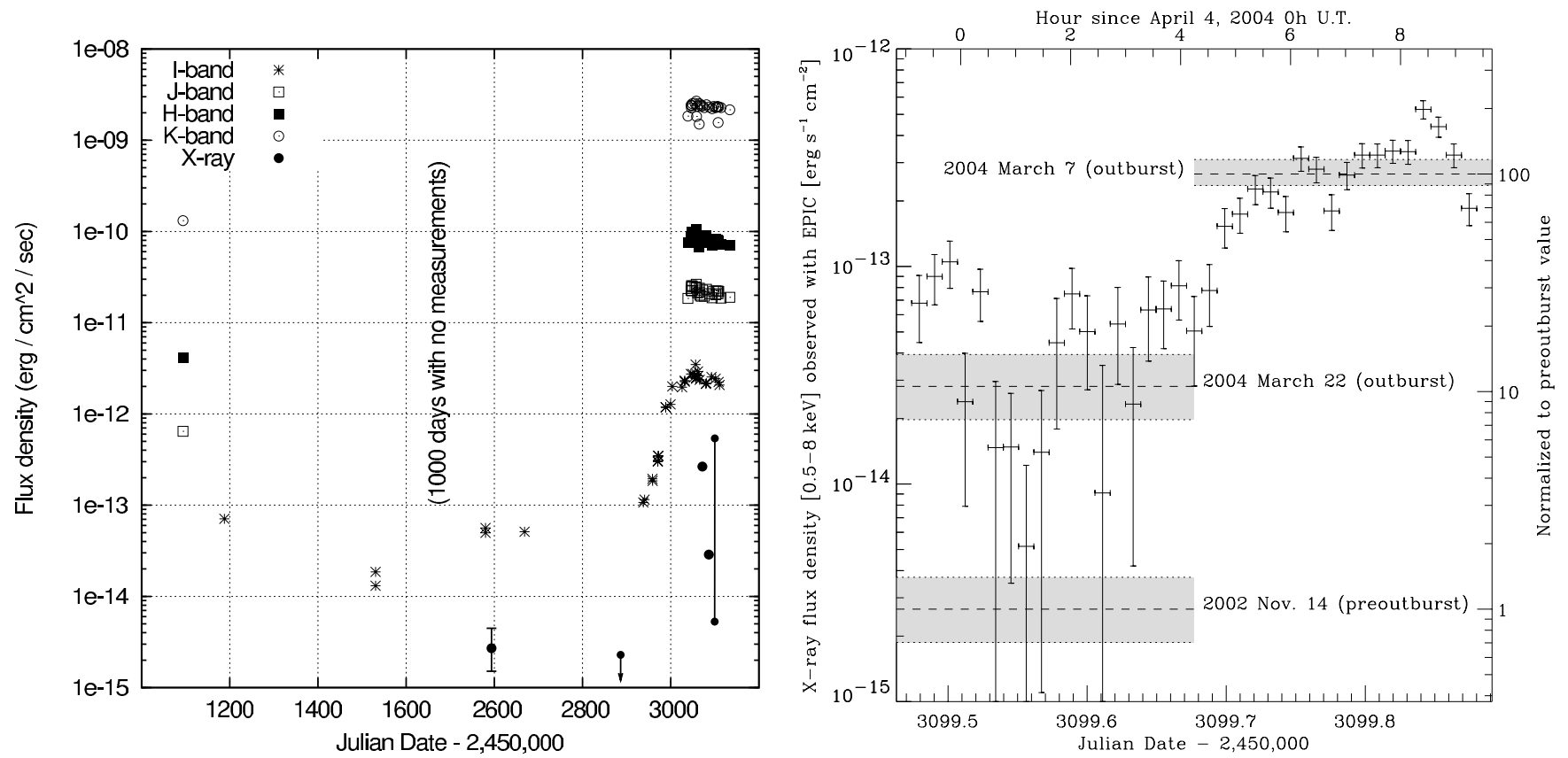

Fig. 7. Long term multiwavelength variation of the observed flux density of V1647 Ori. The left hand panel (adapted from Kastner et al. 2004) shows the variation of the observed flux density of V1647 Ori from the optical ( $I$ band), near-IR ( $J, H$, and $K$ bands), and X-ray ( $0.5-8 \mathrm{keV}$ energy band). The first three X-ray measurements are from Chandra (Kastner et al. 2004), where we have computed for the 2002 November 14 measurement the $90 \%$ confidence level from observed counts using Gehrels (1986) statistics. The dotted arrow marks the X-ray upper limit at the $90 \%$ confidence level computed from the XMM-Newton observation of HH24-26 on 2003 September 3 (Ozawa et al., in preparation). The range of X-ray flux density that we report here with XMM-Newton is represented by a segment, detailed in the right hand panel. The right hand panel shows the X-ray flux density observed with XMM-Newton, computed from the EPIC light curve (upper panel of Fig. 2) using a conversion factor from count rate to flux density derived from our best spectral fit (Fig. 5). One sigma error bars are given for both XMM-Newton and Chandra fluxes.
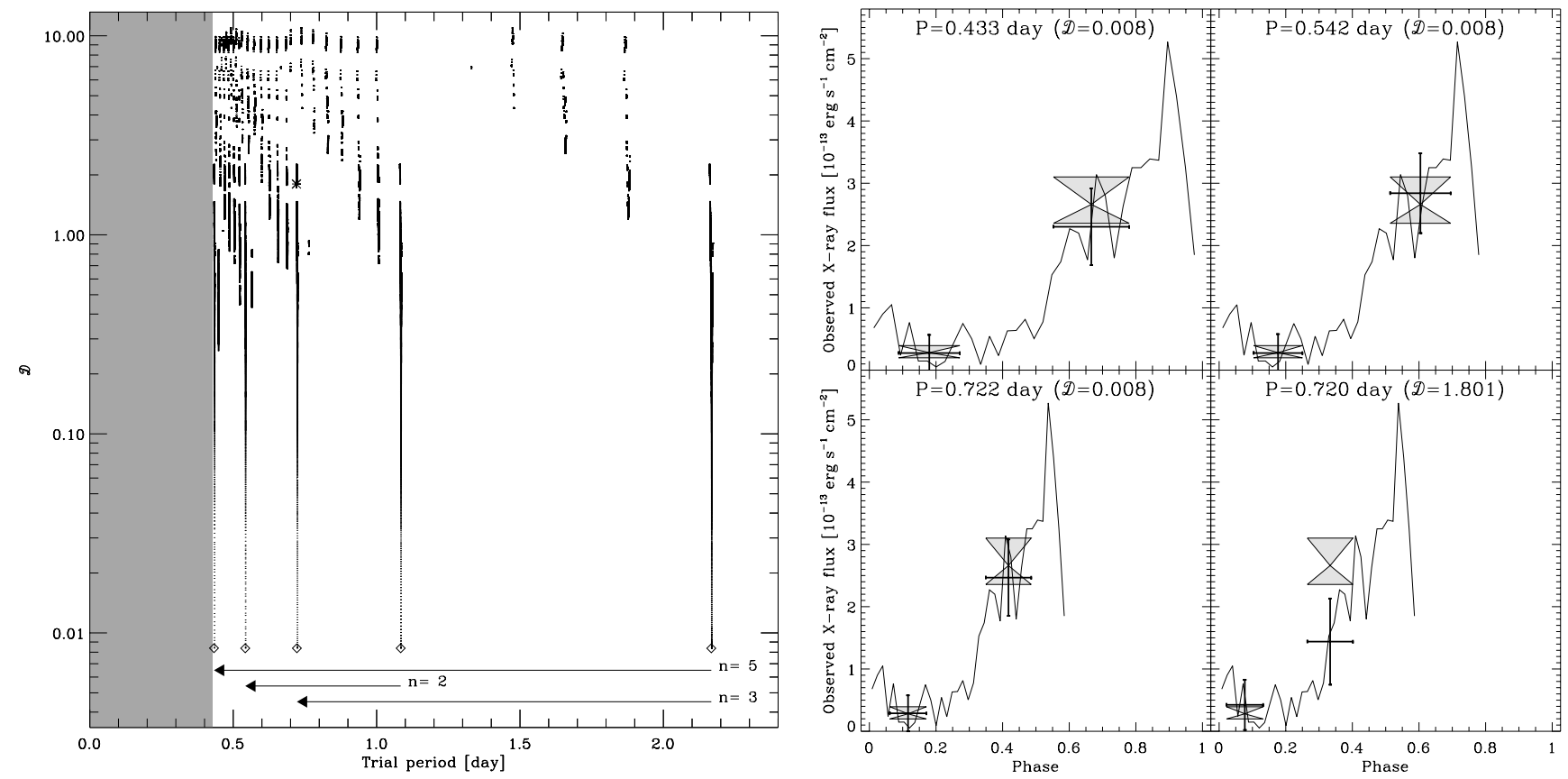

Fig. 8. Folded XMM-Newton and Chandra X-ray fluxes of V1647 Ori. The left hand panel plots the function $\mathcal{D}$ versus trial period, from the value given by the length of the XMM-Newton observation to 2.3 days with period step of $10^{-6} \mathrm{~s}$. This function is defined only for periods allowing the folding of the two Chandra measurements with the XMM-Newton light curve (see text for details). Diamonds mark the best three shortest trial periods with their corresponding $n^{\text {th }}$-harmonic. The right hand panel displays the resulting XMM-Newton (line) and Chandra (grey hourglasses) flux measurements folded using these three periods. The error bars represent the equivalent XMM-Newton flux integrated during the Chandra observing time. The last plot of the right hand panel shows for comparison a bad fit corresponding to the point marked by an asterisk in the left hand panel. 
where $P$ is the trial period; $F_{C X O, i}$ is one of the two Chandra flux measurements; $\tilde{F}_{X M M, i, P}$ is the XMM-Newton flux integrated over the Chandra observational window (depending of the trial period) using a linear interpolation of the $X M M-N e w t o n$ data points; $\sigma_{X M M, i, P}$ is the standard deviation of the XMM-Newton flux in the Chandra observational window. This function is defined only for periods allowing the folding of the two Chandra measurements with the XMM-Newton light curve. $\mathcal{D}$ defines in the folded light curve the quadratically averaged distance between Chandra measurements and the $X M M$-Newton light curve, in units of the standard deviation of the XMM-Newton flux in the Chandra observational window. We computed numerically $\mathcal{D}$ versus trial periods, from the value given by the length of the XMM-Newton observation to 2.3 days, with period step of $10^{-6} \mathrm{~s}$. The left hand panel of Fig. 8 shows the resulting value of $\mathcal{D}$ for each trial period. The structure of the $\mathcal{D}$ function thus obtained is non continuous and cyclical, thereby appearing similar to an attractor. We consider the periods providing the smallest value of $\mathcal{D}$ as the best period candidates. The first three period candidates are: $P=0.43$, 0.54 , and 0.72 day, with $\mathcal{D} \sim 0.008$. The other periods leading to a minimum value for $\mathcal{D}$ are $n^{\text {th }}$-harmonics (where $n$ is an integer) of these first periods. The right hand panel of Fig. 8 shows the corresponding X-ray folded light curves obtained with these period candidates. The last plot in Fig. 8 illustrates a typical period-folding result when $\mathcal{D}$ is greater than 1 , corresponding to the asterisk point in the left hand panel of Fig. 8; clearly, in this case, we obtain a poor fit. Because our combined Chandra and XMM-Newton dataset spans nearly 1 month, any folding of the Chandra flux measurements with periods much smaller than $\sim 1$ month is very sensitive to the assumed value of the period.

From both an observational and a physical point of view, the 0.72 day period appears the most likely. Observationally, with this period, roughly $60 \%$ of the phase is covered by the $X M M-N e w t o n$ observation, where during half of this time interval the X-ray flux is close to one of the two levels measured with Chandra. Of course if we assume that the light curve is indeed symmetric about phase 0.5 , then the X-ray flux is, during the whole period, close to one of the two levels measured with Chandra precisely half the time. Therefore random short X-ray observations would catch easily one of these two characteristic level of activity, explaining naturally the results obtained with Chandra. Physically, this period corresponds to the time scale of Keplerian rotation at a distance of 3.4 solar radius from a one solar mass star, or equivalently to a distance of 1 (1.4) stellar radius for a one solar mass star with an age of 0.5 (1.0) Myr (Palla \& Stahler 1999).

\section{Discussion}

Until very recently, no X-ray satellites have had the opportunity to observe a PMS eruptive object at the beginning of its outburst. In this respect the recent Chandra and XMM-Newton observations of V1647 Ori offer new insight into a PMS accretion burst and, hence, into PMS accretion processes. During phases of normal accretion activity in T Tauri stars $\left(\dot{M}_{\mathrm{acc}} \sim 10^{-7} M_{\odot} \mathrm{yr}^{-1}\right)$ the dynamic pressure from the disk accretion flow is balanced by the magnetic pressure of the stellar magnetosphere at the Alfvén radius (Ghosh \& Lamb 1978; Koenigl 1991). The magnetic pressure inside this radius (close to the co-rotation radius) is strong enough to prevent the accreting material in the disk midplane from spiralling towards the star, but rather lifts it into free fall along the stellar field lines, leading to magnetospheric accretion (see review by Shu et al. 2000).

In contrast, during an accretion outburst, the ram pressure from the accretion disk flow is sufficient to push the inner edge of the accretion disk within the co-rotation radius, leading to strong magnetic interaction between the star magnetosphere and its inner accretion disk. This interaction area, called the reconnection ring, is then a possible source of hard and soft $\mathrm{X}$-ray emission from magnetic reconnection events (Shu et al. 1997). Strong reconnection events will also occur when differential rotation between the inner disk edge and stellar magnetospshere cause fields lines to twist, strengthen and expand (Lovelace et al. 1995; Goodson et al. 1997; Matt et al. 2002). In these cases reconnection drives outflows occuring periodically above and below the disk (Goodson \& Winglee 1999; Goodson et al. 1999).

In the most extreme case, the accretion flow is even sufficient to crush the stellar magnetic field against the stellar photosphere, leading to the formation of a boundary layer at the inner edge of the accretion disk (Hartmann 1998), which may quench the star-disk X-ray emission.

The previous shorter (5 ks exposures) observations of V1647 Ori obtained with Chandra have shown on 2004 March 7 during the outburst an enhanced X-ray flux compared to the pre-outburst level measured by Chandra on 2002 November 14; and a drop of the X-ray flux on 2004 March 22, which we previously interpreted as the possible onset of a quenching X-ray emission phase, or the triggering of a phase of strong variability in both X-ray luminosity and temperature (Kastner et al. 2004). The longer 38 ks observation with XMM-Newton of V1647 Ori on 2004 April 7, presented here, shows that there is indeed as yet no quenching X-ray emission phase, but there is clearly enhanced X-ray variability.

The detailed X-ray spectrum obtained with XMM-Newton improves of our knowledge of the X-ray properties during the outburst phase, showing that $\sim 75 \%$ of the intrinsic X-ray emission in the $0.5-8 \mathrm{keV}$ energy band may be explained by an accretion shock onto the photosphere of this low-mass star, whereas $\sim 25 \%$ of the total X-ray emission comes from a plasma heated by a magnetic reconnection mecanism. The dramatic increase of accretion rate, producing the optical/IR outburst, may also explain the observed rise of X-ray emission from V1647 Ori: first by increasing the quantity of gas falling onto the stellar surface, which radiates soft X-rays at the accretion shock; second by pushing the inner accretion disk boundary inside the corotation radius, which would increase the contribution of the reconnection ring to the soft/hard X-ray emission.

Our quantile analysis has shown that large increase of the count rate is not associated with any large temperature variations as for typical X-ray flares from YSOs, suggesting that 
we are likely also observing variations of the emission measure. The variation of the emission measure can be produced by a variation of the (observed) plasma volume and/or a variation of the plasma electronic density. We cannot totally exclude that we observed an unusual X-ray flare with a strong variation with time of the plasma electronic density in the flaring loop. However, the comparison of our XMM-Newton light curve with the Chandra measurements has suggested that the X-ray flux of V1647 Ori could be periodic, with a likely period of 0.72 day - corresponding to the Keplerian rotation of close material in the close vicinity of the star. Therefore, the enhanced X-ray variability of V1647 Ori is more likely explained by a variation of the observed plasma volume by rotational modulation. In this scenario, the inner part of the accretion disk eclipses periodically a fraction of the plasma volume close to the stellar surface, producing a dimming of the observed X-ray flux by a factor of about 20. Rotational modulation by accretion funnels has been proposed to explain optical eclipses observed in the classical T Tauri star AA Tau, where the accretion disk is thought to be warped by the magnetospheric magnetic dipole inclined respectively to the star's spin (Bouvier et al. 1999).

We stress that the optical/IR flux of V1647 Ori is dominated by the outburst luminosity of its inner accretion disk and scattered light from the surrounding nebulosity, therefore any opti$\mathrm{cal} / \mathrm{IR}$ modulations of the photospheric flux are difficult if not impossible to observe. Therefore X-rays, likely produced only close to the central star, offer a unique observational window to probe the star-disk interaction area during this PMS evolutionary phase in which the accretion rate, and hence the source luminosity, are dramatically elevated above normal.

Longer X-ray observations with XMM-Newton and Chandra will be needed to confirm both the candidate period, and to validate the scenario proposed here to explain the enhanced X-ray variability.

Acknowledgements. We would like to thank the Target of Opportunity panel of XMM-Newton and the XMM-Newton's project scientist Norbert Schartel, who gave us the opportunity to observe V1647 Ori during its outburst. We thanks the anonymous referee for his comments and suggestions. H.O. acknowledges the support of the Conseil National des Astronomes et Physiciens. Based on observations obtained with the XMM-Newton, an ESA science mission with instruments and contributions directly funded by ESA member states and the USA (NASA). We used archival acquisition image made with ESO Very Large Telescope at Paranal Observatory under programme ID 272.C-5045.

\section{References}

Ábrahám, P., Kóspál, Á., Csizmadia, S., et al. 2004, A\&A, 419, L39 Andrews, S. M., Rothberg, B., \& Simon, T. 2004, ApJ, 610, L45

Anthony-Twarog, B. J. 1982, AJ, 87, 1213

Aspin, C., \& Reipurth, B. 2004, IAU Circ., 8396, 3

Bouvier, J., Chelli, A., Allain, S., et al. 1999, A\&A, 349, 619

Briceño, C., Vivas, A. K., Hernández, J., et al. 2004, ApJ, 606, L123

Cardelli, J. A., Clayton, G. C., \& Mathis, J. S. 1989, ApJ, 345, 245

Clark, F. O. 1991, ApJS, 75, 611

Eislöffel, J., \& Mundt, R. 1997, AJ, 114, 280

Gehrels, N. 1986, ApJ, 303, 336

Ghosh, P., \& Lamb, F. K. 1978, ApJ, 223, L83
Goodson, A. P., Winglee, R. M., \& Boehm, K. 1997, ApJ, 489, 199

Goodson, A. P., \& Winglee, R. M. 1999, ApJ, 524, 159

Goodson, A. P., Böhm, K., \& Winglee, R. M. 1999, ApJ, 524, 142

Hartmann, L., \& Kenyon, S. J. 1996, ARA\&A, 34, 207

Hartmann, L. 1998, Accretion processes in star formation (Cambridge: Cambridge University Press)

Herbig, G. H. 1966, Vistas in Astronomy, 8, 109

Herbig, G. H. 1977, ApJ, 217, 693

Herbig, G. H., Aspin, C., Gilmore, A. C., Imhoff, C. L., \& Jones, A. F. 2001, PASP, 113, 1547

Hong, J., Schlegel, E. M., \& Grindlay, J. E. 2004, ApJ, 614, 508

Imanishi, K., Koyama, K., \& Tsuboi, Y. 2001, ApJ, 557, 747

Imanishi, K., Nakajima, H., Tsujimoto, M., Koyama, K., \& Tsuboi, Y. 2003, PASJ, 55, 653

Jansen, F., Lumb, D., Altieri, B., et al. 2001, A\&A, 365, L1

Kaastra, J. S., Mewe, R., \& Nieuwenhuijzen, H. 1996, in UV and X-ray Spectroscopy of Astrophysical and Laboratory Plasmas, ed. K. Yamashita, \& T. Watanabe (Tokyo: Univ. Acad. Press), 411

Kastner, J. H., Huenemoerder, D. P., Schulz, N. S., Canizares, C. R., \& Weintraub, D. A. 2002, ApJ, 567, 434

Kastner, J. H., Richmond, M., Grosso, N., et al. 2004, Nature, 430, 429

Kenyon, S. J., \& Hartmann, L. W. 1991, ApJ, 383, 664

Koenigl, A. 1991, ApJ, 370, L39

Kun, M., Acosta-Pulido, J. A., Moor, A., et al. 2004, A\&A, submitted [arXiv: astro-ph/0408432]

Lis, D. C., Menten, K. M., \& Zylka, R. 1999, ApJ, 527, 856

Lovelace, R. V. E., Romanova, M. M., \& Bisnovatyi-Kogan, G. S. 1995, MNRAS, 275, 244

Lupton, R., Blanton, M. R., Fekete, G., et al. 2004, PASP, 116, 133

Mallas, J. H., \& Kreimer, E. 1978 (Cambridge: Sky Publication Co), Mass

Mason, K. O., Breeveld, A., Much, R., et al. 2001, A\&A, 365, L36

Matt, S., Goodson, A. P., Winglee, R. M., \& Böhm, K. 2002, ApJ, 574,232

McGehee, P. M., Smith, J. A., Hende, A. A., et al. 2004, ApJ, 616, 1058

McLaughlin, D. B. 1946, AJ, 52, 109

McNeil, J. W. 2004, IAU Circ., 8284, 1

Mitchell, G. F., Johnstone, D., Moriarty-Schieven, G., Fich, M., \& Tothill, N. F. H. 2001, ApJ, 556, 215

Morrison, R., \& McCammon, D. 1983, ApJ, 270, 119

Muzerolle, J., Megeath, S. T., Flaherty, K. M., et al. 2005, ApJ, 620, L107

Palla, F., \& Stahler, S. W. 1999, ApJ, 525, 772

Reipurth, B., \& Aspin, C. 2004, ApJ, 606, L119

Rettig, T. W., Brittain, S. D., Gibb, E. L., Simon, T., \& Kulesa, C. A. 2004, ApJ, in press

Samus, N. N. 2004, IAU Circ., 8354, 1

Shu, F. H., Shang, H., Glassgold, A. E., \& Lee, T. 1997, Science, 277, 1475

Shu, F. H., Najita, J. R., Shang, H., \& Li, Z.-Y. 2000, Protostars \& Planets IV, 789

Simon, T., Andrews, S. M., Rayner, J. T., \& Drake, S. A. 2004, ApJ, 611,940

Strüder, L., Briel, U., Dennerl, K., et al. 2001, A\&A, 365, L18

Tsuboi, Y., Koyama, K., Murakami, H., et al. 1998, ApJ, 503, 894

Turner, M. J. L., Abbey, A., Arnaud, M., et al. 2001, A\&A, 365, L27

Vacca, W. D., Cushing, M. C., \& Simon, T. 2004, ApJ, 609, L29

Vuong, M. H., Montmerle, T., Grosso, et al. 2003, A\&A, 408, 581

Walter, F. M., Stringfellow, G. S., Sherry, W. H., \& Pollatou, A. F. 2004, AJ, 128, 1872 